\title{
Nanopore Integrated Nanogaps for DNA Detection
}

\author{
Axel Fanget, ${ }^{\dagger}$ Floriano Traversi, ${ }^{\ddagger}$ Sergey Khlybov, ${ }^{\ddagger}$ Pierre Granjon, ${ }^{\ddagger}, \S$ Arnaud Magrez, ${ }^{\dagger}, \|$ László Forró, ${ }^{*}{ }^{\dagger}$ \\ and Aleksandra Radenovic* $*$ \\ ${ }^{\dagger}$ Laboratory of Physics of Complex Matter, School of Basic Sciences and ${ }^{\ddagger}$ Laboratory of Nanoscale Biology, Bioengineering Institute, \\ School of Engineering, EPFL, 1015 Lausanne, Switzerland \\ ${ }^{\S}$ Grenoble Image Speech Signal Automatics Laboratory, Grenoble Institute of Technology, Grenoble, France \\ "Crystal Growth Facility, School of Basic Sciences, EPFL, 1015 Lausanne, Switzerland
}

\section{Supporting Information}

ABSTRACT: A high-throughput fabrication of sub-10 nm nanogap electrodes combined with solid-state nanopores is described. These devices should allow concomitant tunneling and ionic current detection of translocating DNA molecules. We report the optimal fabrication parameters in terms of dose, resist thickness, and gap shape that allow easy reproduction of the fabrication process at wafer scale. The device noise and current voltage characterizations performed and the influence of the nanoelectrodes on the ionic current noise is identified. In some cases, ionic current rectification for connected or biased
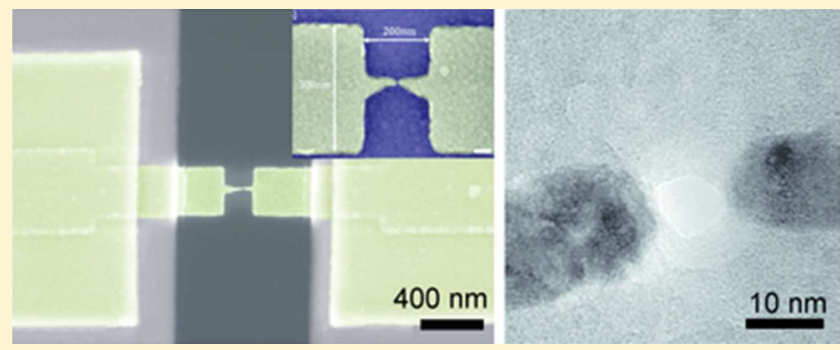
nanogap electrodes is also observed. In order to increase the extremely low translocation rates, several experimental strategies were tested and modeled using finite element analysis. Our findings are useful for future device designs of nanopore integrated electrodes for DNA sequencing.

KEYWORDS: Nanopore, DNA, nanogaps, single molecule

T $\mathrm{n}$ 2006, Lagerqvist et al. ${ }^{1}$ proposed novel modality of 1 nanopore sequencing that exploits quantum mechanical phenomena of electron tunneling allowing identification of individual bases. At the heart of this concept are nanochannels or nanopores that are required to spatially confine DNA molecules whereas two electrodes separated by a few nanometers act as tunneling probes. Since the tunneling current is exponentially decaying with distance, the device is highly sensitive to the size, shape, and orientation of the molecules residing in the nanogap. Thanks to this property, a signal specific to the base residing in the nanogap will not interfere with the signals from neighboring bases. ${ }^{2}$ This approach overcomes nanopore spatial resolution limit imposed by the typical silicon nitride membrane thickness of $10-50 \mathrm{~nm}$, through which it is impossible to distinguish signals in ionic current between adjacent nucleotides on the DNA strand. Although the thickness of membrane embedding the nanopore was recently addressed by using a $0.34 \mathrm{~nm}$ thick graphene sheet, ${ }^{3-5}$ DNA sequencing using direct ionic current measurements has not been fully realized. Transverse electron tunneling using nanoelectrodes on nanopore platform therefore remains a very promising approach. Tsutsui et al. were the first to use sub$2 \mathrm{~nm}$ gold nanoelectrodes based on mechanical break junctions to identify individual nucleotides at concentrations of $5 \mu \mathrm{M}^{6}$ Using suspended nanogap electrodes in distilled water solution, they showed that electron transport occurs by tunneling through nucleotides when freely diffusing individual bases get trapped between the nanogap electrodes. They confirm theoretical predictions ${ }^{1,2}$ that the electrical conductances of the four different bases are significantly different. In a follow-up experiment, they embedded nanogap electrodes into a planar $\mathrm{SiO}_{2}$ layer, resulting in an in-plane microchannel formed by the nanogap itself. ${ }^{7}$ In a nanochannel, they could similarly recognize mononucleotides and short DNA oligomers of up to 22 bases by tunneling measurements. However, further investigations are needed to electrophoretically drive longer molecules and verify their results by concomitant monitoring of ionic current signals. To our knowledge, only few experimental reports show fabrication of nanopores with embedded tunneling electrodes that should permit concomitant monitoring of both signals. Gierhart et al. $^{8}$ were first to report a prototype of the device able to detect gold nanoparticles. However, their fabrication method yielded $20 \mathrm{~nm}$ large nanogaps and presented limited evidence of tunneling detection of nanoparticles. In a more recent paper, Ivanov et al. ${ }^{9}$ use electron beam-induced deposition (EBID) to fabricate tunneling electrodes aligned with nanopores. Although this work presents for the first time concurrent tunneling detection and ionic current detection of DNA molecules in a nanopore platform, it is possible that in the absence of nanoelectrode insulation and at the low sampling frequency $(5 \mathrm{kHz})$ recorded signals might correspond to nonspecific DNA absorptions on

Received: October 15, 2013

Revised: November 26, 2013

Published: December 6, 2013 
a)
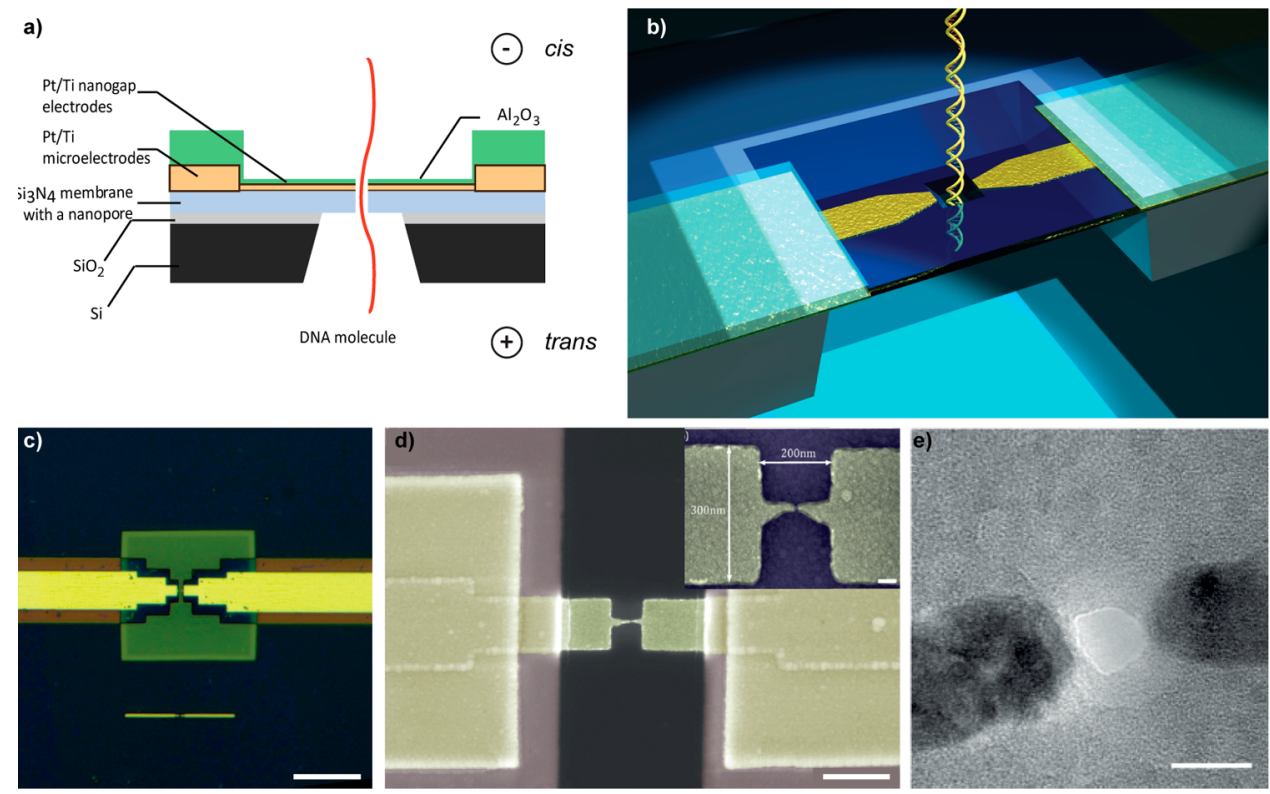

Figure 1. Schematics and fabrication of nanopore/nanogap device. (a) Schematic drawing of our setup (side view). A single DNA molecule is translocating through a nanopore fabricated in a $\mathrm{SiN}_{x}$ membrane. Schematic drawing shows location of the nanogap electrodes made in $\mathrm{Pt} / \mathrm{Ti}$ and different thickness for alumina $\left(\mathrm{Al}_{2} \mathrm{O}_{3}\right)$ layers deposited on nanogap 5 and $150 \mathrm{~nm}$ deposited on the microelectrodes. (b) Artistic representation of the device. (c) Optical micrograph of Pt/Ti nanogap electrodes located in the center of $20 \mu \mathrm{m} \mathrm{SiN}{ }_{x}$ membrane. Picture dimensions $60 \mu \mathrm{m} \times 60 \mu \mathrm{m}$. (d) Colored SEM micrograph of typical nanogap electrodes used for experiments. (e) TEM micrograph of a nanopore drilled between two nanoelectrodes that form nanogap. Micrographs shown in $(\mathrm{c}-\mathrm{e})$ are displaying the same device and scale bars are $10 \mu \mathrm{m}, 400 \mathrm{~nm}$, and $10 \mathrm{~nm}$ respectively.

the metallic nanoelectrodes rather than actual tunneling current. In addition, authors do not provide scanning electron microscope (SEM) or transmission electron microscope (TEM) image of device prototype but similar devices that have been realized at thinner membranes.

The most recent experimental contribution to the field by Healy and colleagues, ${ }^{10}$ describes nanogap electrodes fabrication process by electron beam assisted ablation lithography in a TEM microscope and discusses the importance of the passivation of the electrodes. The paper objectively reports on difficulties to obtain DNA translocations due to device failures.

Here we describe the high-throughput fabrication of sub-10 $\mathrm{nm}$ nanogap electrodes combined with solid-state nanopores that should allow concomitant tunneling and ionic current detection of DNA molecules. The method described here allows fast wafer scale fabrication of sub-10 nm nanogap electrodes with a very high reproducibility. $85 \%$ of the nanogaps of a single wafer ( 72 devices) are smaller than 10 $\mathrm{nm}$, although, depending on the metal layer thickness, nanogaps as small as $7 \mathrm{~nm}$ can be easily found (Supporting Information Figure S2). The fabrication of devices with passivated metallic nanogap electrodes on a suspended $\mathrm{Si}_{3} \mathrm{~N}_{4}$ membrane can be either performed at the single device or fullwafer scale. Fabrication procedure involves four successive steps of electron beam lithography (EBL): (i) definition of metallic nanogap electrodes, (ii) contacting electrodes and pads, (iii) electrodes passivation, and (iv) membrane fabrication. These steps can be performed on wafer scale. Drilling of the nanopores in a transmission electron microscope (TEM) follows and is performed at chip scale. Optical, SEM, and TEM images of a typical device are shown in Figure 1.

To identify the optimal fabrication parameters in terms of dose, resist thickness, and nominal gap size, we proceeded by patterning 2D arrays of nanogaps with variable gap size and dose variation on test wafers. Triangular electrodes are designed to take advantage of proximity effects in EBL (for details on geometry optimization see Supporting Information). For different resist thicknesses, we identify desired nanogap dimension during SEM imaging and associate it with optimal parameters that are then used further in fabrication. In our study, a PMMA thickness of about $50 \mathrm{~nm}$ was found to be optimal. With thicker resist layers, the overall nanogap dimensions quickly increase, while thinner resist layers are limiting the metal layer thickness that can be deposited. We choose following EBL parameters for nanogap definition: beam step size of $1.25 \mathrm{~nm}$ at a dose of $41000 \mu \mathrm{C} / \mathrm{cm}^{2}$ in single pixel line exposure. As detailed in Supporting Information, the optimal designed nanogap size of $25 \mathrm{~nm}$ is very important parameter, since by overexposing and taking advantage of SE scattering sub-10 nm nanogaps are formed.

Next, the optimal thickness of metallic films for nanoelectrodes was investigated. Platinum $(\mathrm{Pt}$ ) was chosen for its high electronic mobility and preferred against gold to minimize surface diffusion at moderate temperatures, typically during successive baking. A $8 \mathrm{~nm}$ Pt film yields the smallest nanogaps with the best reproducibility in terms of nanogap size and conductivity while, thinner films result in high electrode resistances. For thicker films, it is likely that despite sub-10 $\mathrm{nm}$ gaps that can be defined via EBL in the resist, the pattern transfer is not achieved because metallic Pt bridges often remain after lift-off. In contrast to Healy et al., ${ }^{10}$ the microelectrodes contact the nanogaps ones very close to the gap region ensuring the device robustness. Having defined nano- and microelectrodes by e-beam lithography and evaporation, microelectrodes were isolated. The contacting electrodes are next passivated with $150 \mathrm{~nm}$ of $\mathrm{Al}_{2} \mathrm{O}_{3}$, so that thick metallic electrodes are electrochemically inactive, ${ }^{10}$ 

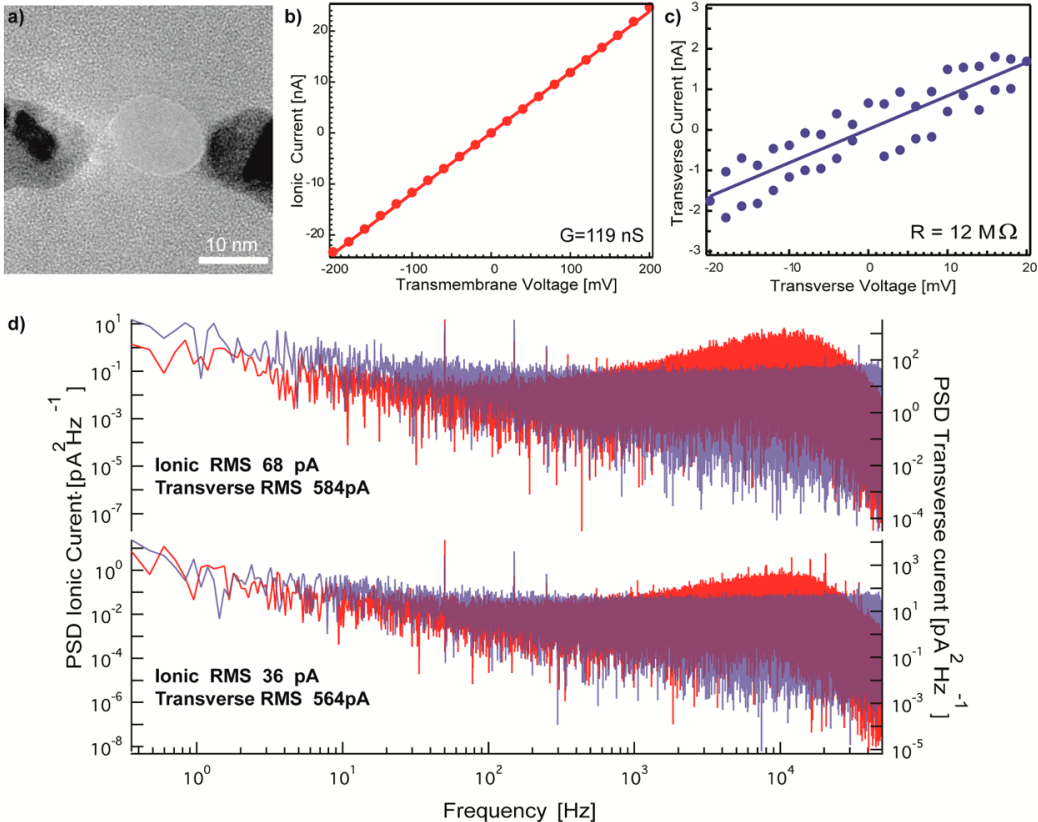

Figure 2. Current voltage and noise characterization of nanopore/nanogap device (a) TEM micrograph of the nanopore which current voltage is shown in (b) and (c). (b) Current voltage characteristic of a nanopore in $1 \mathrm{M} \mathrm{KCl}$. The nanopore is drilled between nanoelectrodes as shown in (a). Dots are experimental points while the continuous line is the fit from which the value of the conductance is extrapolated. (c) Current voltage characteristic of TEM imaged nanogap (shown in panel a) in $1 \mathrm{M} \mathrm{KCl}$ solution. For ideally isolated electrodes, resistance should be G $\Omega$ however we observe $12 \mathrm{M} \Omega$ resistance. Current voltage characteristics are obtained by increasing and decreasing the voltage values. (d) Comparison of the PSD of the ionic current and transverse current of nanopore nanogap device shown in (a), for two situations with the transverse channel disconnected (bottom) and connected and its bias set to $0 \mathrm{mV}$. ( $V_{\mathrm{p}}=0 V_{\mathrm{t}}=$ off (bottom) and $V_{\mathrm{p}}=0$ and $V_{\mathrm{t}}=0$ (top)). One can notice dramatic increase in the RMS for both signals when nanogap is connected.

whereas the thin nanogap area was coated with $6 \mathrm{~nm}$ of $\mathrm{Al}_{2} \mathrm{O}_{3}$ using atomic layer deposition (ALD) so that the nanopore can still be drilled. Finally, a nanopore is drilled by TEM in between of the nanogap electrodes as shown in Figure 1e. Prior to TEM drilling, samples were annealed at $200{ }^{\circ} \mathrm{C}$ to prevent hydrocarbon contamination. ${ }^{11}$ Additional information on the device fabrication process can be found in Supporting Information.

The devices characterization starts first by measuring the ohmic properties of the nanopore with the nanogap electrodes disconnected (floating) from the circuit. Details on microfluidics and data acquisition setup are described in Supporting Information. Figure $1 \mathrm{a}, \mathrm{b}$ schematizes the measurement setup. Nanopore current-voltage characteristics are performed to determine the pore ionic properties that should be linear as shown in Figure $2 \mathrm{~b}$. For $10 \mathrm{~nm}$ large nanopores in $1 \mathrm{M} \mathrm{KCl}$ solution, the obtained conductances range from 10 to $80 \mathrm{nS}$. This range is broader than expected from the model proposed by Kowalczyk et al.. ${ }^{12}$ This is assigned to the presence of the $\mathrm{Al}_{2} \mathrm{O}_{3}$ coating layer allowing better nanopore wetting. ${ }^{13}$ Pores with nonlinear $I-V$ curves within $200 \mathrm{mV}$ were discarded. For pores with ohmic behavior and reasonable conductance, the ionic current at constant voltage was observed stable for long time periods. By integrating the spectral density of the ionic current with respect to the frequency, we calculated typical RMS noise. An $I_{\mathrm{RMSp}}$ of $40 \mathrm{pA}$ in $1 \mathrm{M} \mathrm{KCl}$ (data not shown) is found. It is slightly higher than previously reported values for similar pore sizes. ${ }^{14}$

Next we perform $I-V_{\mathrm{t}}$ sweeps to measure the nanogap resistance with voltage applied on the pore $V_{\mathrm{p}}=0$ in $1 \mathrm{M} \mathrm{KCl}$. Figure $2 \mathrm{c}$ shows typical nanogap current voltage characteristics (where $V_{\mathrm{t}}$ stands for transverse voltage applied on the nanogap, while $V_{\mathrm{p}}$ is voltage applied on the pore). For over 50 devices measured, the different sub-10 $\mathrm{nm}$ nanogap electrodes resistances cover a range between few hundreds of $\mathrm{k} \Omega$ to several tens of $M \Omega$. As the observed variability in the values does not correlate either with the gap distance or with the distance to the nanopore edges, the quality of the insulation layer of $\mathrm{Al}_{2} \mathrm{O}_{3}$ is believed to remain the most critical parameter for the gap resistance. Supporting Information Figure S3b shows a typical transverse current power spectral density (PSD) calculated for a nanogap voltage of $20 \mathrm{mV}$ in the absence of ionic voltage $V_{\mathrm{p}}=0 \mathrm{~V}$. The shape of this PSD clearly highlights the presence of a flicker noise below $50 \mathrm{~Hz}$ (PSD in $1 / f)$, and a white noise above this frequency (constant PSD). In $1 \mathrm{M} \mathrm{KCl}$, the $I_{\mathrm{RMSt}}$ of $570 \mathrm{pA}$ obtained is 1 order of magnitude higher than the noise obtained for the ionic current. An overall increase with frequency of the ionic current noise $I_{\mathrm{RMSp}}$ and transverse nanogap current $I_{\mathrm{RMSt}}$ are observed for all conditions at which we operate the device $V_{\mathrm{p}}>0 \mathrm{~V}$ and $V_{\mathrm{t}}=20 \mathrm{mV}$. As shown in Figure 2d, this increase happens when we connect nanogap electrodes to the circuit, top panel $\left(V_{\mathrm{p}}=0 \mathrm{mV}\right.$ and $V_{\mathrm{t}}=0 \mathrm{mV}$, connected), compared to low noise situations with disconnected nanogap bottom panel $\left(V_{\mathrm{p}}=0 \mathrm{mV}\right.$ and $\left.V_{\mathrm{t}}=0 \mathrm{mV}\right)$. For disconnected nanogap, ionic RMS noise is $36 \mathrm{pA}$ close to the values reported in literature for similar size pore and membrane thickness. No link exists between the ionic and transverse currents with disconnected nanogap electrodes because the spectral coherence stays close to 0 whatever the frequency band. However, a small but significant linear link exists in the high-frequency band $(f>20 \mathrm{kHz})$ with connected electrodes as shown in Supporting Information Figure S4. The increase of RMS noise of both currents may be a sign of a capacitive coupling between the two channels. Interestingly, in some 

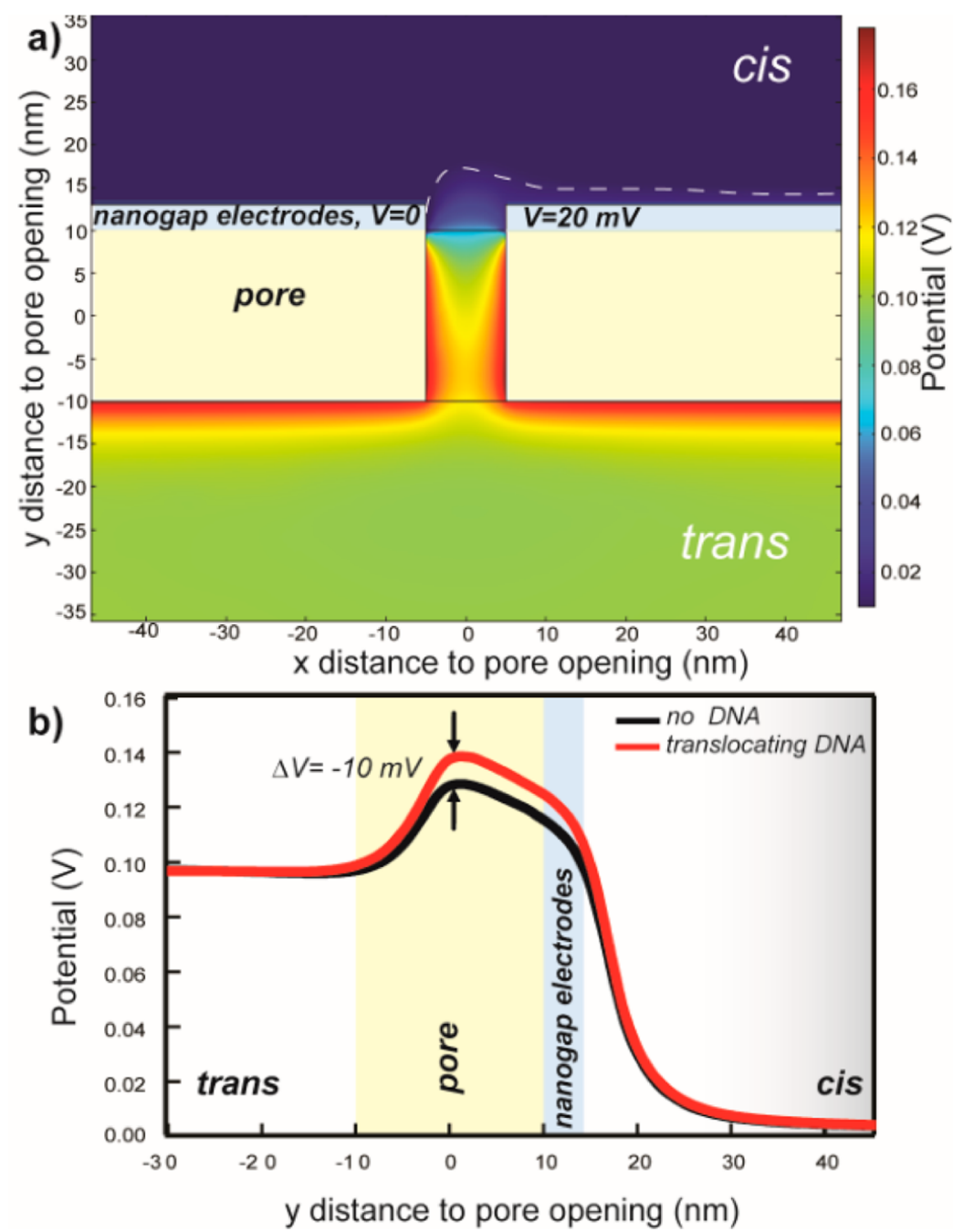

Figure 3. Simulation of the electric potential distribution of the nanopore with nanoelectrodes in two dimensions. Simulations are performed for the experimental conditions presented in Figure 1. (a) A close up view of the electric potential distribution for the experimental conditions when applied field is $100 \mathrm{mV}$ in $10 \mathrm{mM} \mathrm{KCl}$ and no DNA translocates the pore. Here we consider $y<-10 \mathrm{~nm}$, trans side; $-10 \mathrm{~nm}<y<10 \mathrm{~nm}$, nanopore, $y>10$ $\mathrm{nm}$, cis side; $10 \mathrm{~nm}<y<13 \mathrm{~nm}, \mathrm{Pt} / \mathrm{Ti}$ nano electrodes. (b) For two different conditions: pore without DNA and with translocating DNA. Translocating dsDNA is modeled as extended $100 \mathrm{~nm}$ long linear segment. The $20 \mathrm{mV}$ bias is applied on nanogap electrodes. The dashed line points to the asymmetry of the potential distribution.

devices we observed ionic current rectification when the nanogap electrodes were connected and at $V_{\mathrm{t}}=0 \mathrm{~V}$ or biased $V_{\mathrm{t}}$ $\neq 0 \mathrm{~V}$ while the same device exhibits perfect ohmic behavior if measured with disconnected electrodes, as shown in Supporting Information Figure S5. The current rectification does not seem to follow a particular trend with respect to the nanogap bias $V_{\mathrm{t}}$. In the example presented in Supporting Information Figure S5, the rectification factor for the same pore is comprised between 8 and 11. Different behaviors were observed for different nanopores. In some cases, the current quickly reaches a plateau while it sometimes keeps increasing in the limits of the voltage sweep. However, the ionic current response at negative pore bias and in all rectification cases follows the corresponding response obtained with disconnected electrodes while for the positive bias shows rectification behavior.

In nanofluidics systems, asymmetric geometries or nonuniform surface-charges distribution can result in ionic current rectification. ${ }^{13,15-19}$ TEM sculpted nanopores are supposed to exhibit hourglasses shapes, which are symmetric structures. Moreover rectification is not observed when electrodes are disconnected. Therefore, connecting the electrodes must induce a modification of the surface charges that causes rectifying behaviors and in particular favors the mobility of the charge carriers at positive voltage. The mechanism is challenging to understand because the rectification behaves independently of the potential applied to the electrodes and also occurs at 0 bias. Our result differs from the conclusion drawn by Healy et al. who observed that the presence of nanoelectrodes does not affect the ionic current. ${ }^{10}$

A noise and current voltage characterization of each nanogap-nanopore device is performed prior to any DNA translocation experiments. For such experiments, we considered only pores with linear current voltage characteristics and $I_{\mathrm{RMSp}}$ and $I_{\mathrm{RMSt}}$ having values comparable to the device presented in Figure $2 \mathrm{~b}$. As shown in Figure 1a, the DNA is flushed through the cis chamber at a concentration of $50 \mu \mathrm{g} /$ $\mathrm{mL}$. Next, a positive potential is applied to the membrane and the DNA molecules should translocate from the cis to the trans side of the membrane. For transverse sensing, a positive potential of $20 \mathrm{mV}$ is applied between the nanogap electrodes. In order to preserve the quality of the insulation layer, we avoided application of a higher nanoelectrode bias. The main 

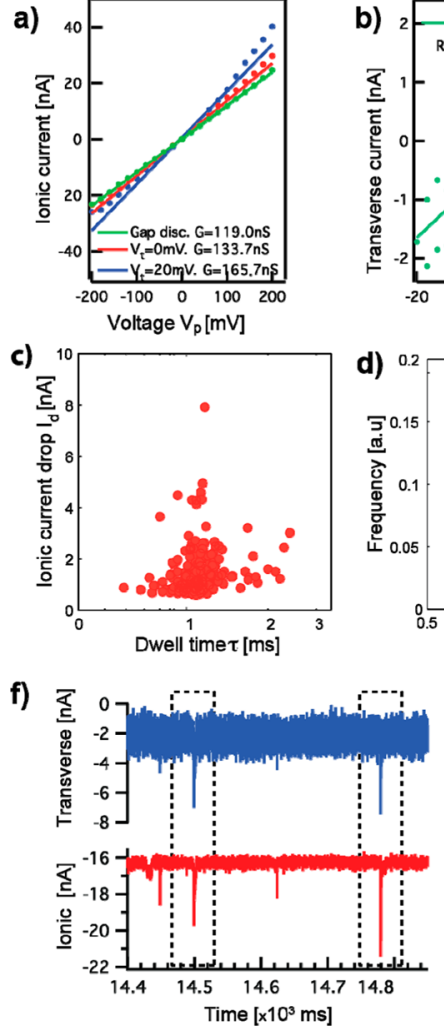
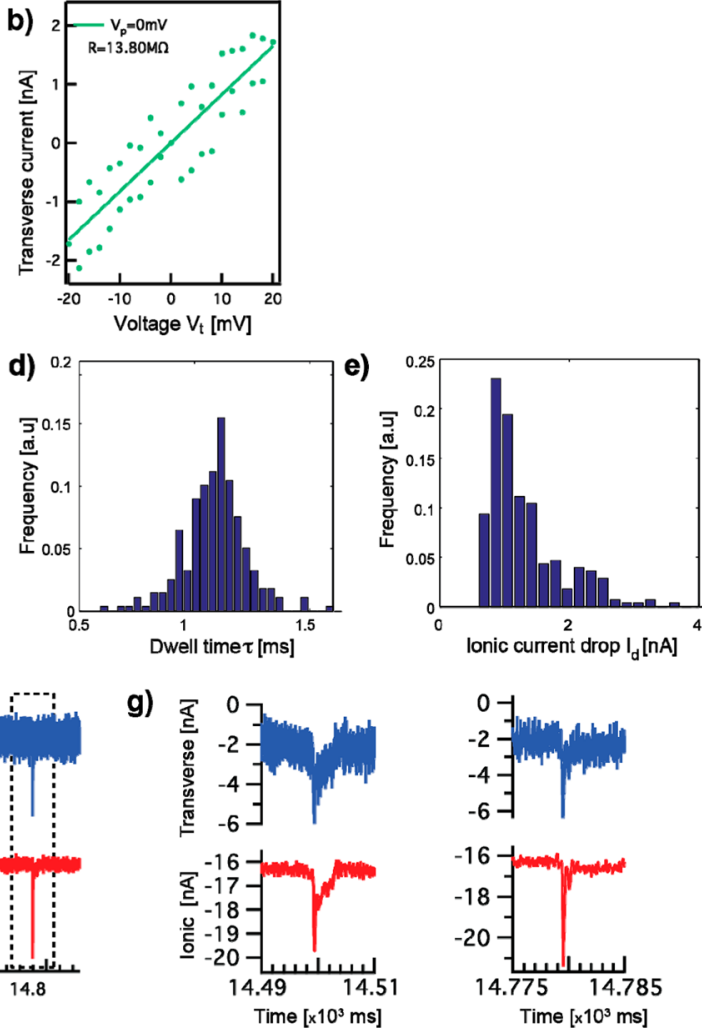
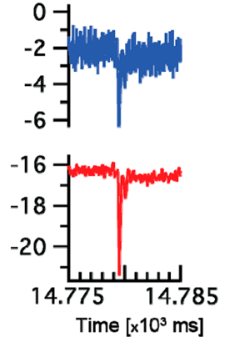

Figure 4. Simultaneous detection of translocation events in ionic and transverse current traces. (a,b) $I-V$ properties of the ionic and transverse current in $1 \mathrm{M} \mathrm{KCl}$. (c) Dwell time and drop amplitude of the ionic current events as detected for Hind III digested $\lambda$. (d,e) Dwell times and amplitudes distributions analysis. $(\mathrm{f}, \mathrm{g})$ Example of correlated events in the ionic channel (red) and transverse channel (blue) recorded during DNA translocations.

experimental issue we experienced is the extreme difficulty of detecting DNA translocations. In order to make sure that the observed lack of translocation events was not a detection issue, the presence of DNA molecules in the trans reservoir was tested several times by polymerase chain reaction (PCR) after experiments. All tests turned out as negative. In addition, control devices were fabricated on all wafers, which consist of a bare nanopore with typical sizes of $8 \times 10 \mathrm{~nm}$ through membranes of similar size and thickness as used for nanogap devices. As expected, numerous translocation events could be detected as shown in Supporting Information Figure S6. At this point, two standard approaches that allow increased translocation rate were tried. ${ }^{20,21}$ First the ionic transmembrane potential was gradually increased ${ }^{20}$ up to $800 \mathrm{mV}$ with no apparent effect on translocation rate. Next, we tested salt gradient conditions that should lead to increased translocation rate as recently proposed by Wanunu et al.. ${ }^{21}$ To increase the capture rates and obtain better statistics on the number of events, salt gradient was established by placing the nanogap electrodes in the cis chamber where the ionic strength was kept at $10 \mathrm{mM} \mathrm{KCl}$ while the concentration in the trans chamber has been increased to $100 \mathrm{mM} \mathrm{KCl}$. The new experimental geometry is schematized in Supporting Information Figure S7a. Having nanoelectrodes in lower ionic strength solutions should be beneficial for tunneling detection once nanoelectrodes are separated by less than $5 \mathrm{~nm}$ and it should preserve the quality of the insulation layer since lower $\mathrm{KCl}$ ionic strength lowers the solution electrochemical activity. When working in salt gradient and high transmembrane bias voltage conditions $V_{\mathrm{p}}>500 \mathrm{mV}$, the capture rate follows Van't Hoff-Arrhenius relationship
$R_{\text {capture }} \propto e^{\mathrm{V} 22}$ and a theoretical 20-fold increase in the capture rate compared to previous experimental conditions is expected. Unfortunately, this effect has not been observed. Opposite configuration with nanogap electrodes located in trans side was tested. It similarly resulted in sparse or no translocation for numerous devices Supporting Information Figure S7b.

In order to gain better insights into the ion transport mechanisms inside a nanopore surrounded by nanogap electrodes, we performed finite element analysis simulations of the potential distributions near the pore for several experimental conditions and device geometries, that is, nanogap electrodes either on cis or trans side of the device. The electric potential distribution was modeled in the presence and absence of the translocating DNA molecule. On the basis of obtained COMSOL simulations, the optimal experimental geometry for concomitant translocation detection is shown in Figure 3 where the nanogap electrodes are located on the cis side of the device. Here the translocating DNA molecule results in a potential change of $\Delta V=-10 \mathrm{mV}$. Note that this high potential change should be detected with transverse electrodes even in conditions that do not allow tunneling detection, such as high salt concentration and for nanogap electrodes spaced by more than $3 \mathrm{~nm}$. For the device with electrodes at the trans side potential change is also $\Delta V=10 \mathrm{mV}$ as shown in Supporting Information Figure S8. Although we took great care of device modeling, fabrication and characterization by optimizing experimental geometry and by using only high quality devices that showed ohmic behavior and low noise, only few DNA translocations were detected. However, when sparsely present, DNA translocations were simultaneously detected in ionic and 
transverse current as shown in Figure 4. Although, our modeling suggest that optimal conditions are reached for concentration gradient (Figure 3), the data presented in Figure 4 have been taken in standard $1 \mathrm{M} \mathrm{KCl}$ conditions and analyzed using OpenNanopore software. ${ }^{23,24}$ Presented results show that even for sub-10 nm sized nanogaps the current detected using the "tunneling" electrodes at $1 \mathrm{M} \mathrm{KCl}$ originates solely from ionic current changes. In addition, at $1 \mathrm{M} \mathrm{KCl} \mathrm{effect}$ of potential modulation Supporting Information Figure S9 is much smaller compared to conditions presented in Figure 3 and Supporting Information Figure S8 . Therefore, obtaining tunneling detection on nanopore platform will require invention of fabrication techniques that will allow highthroughput sub-2.5 $\mathrm{nm}$ nanogap production ${ }^{25}$ and operation at low salt conditions.

To conclude we present high-throughput fabrication of sub$10 \mathrm{~nm}$ nanogap electrodes combined with solid-state nanopores that allowed concomitant tunneling and ionic current detection of translocating DNA molecules. We report sparse DNA translocations for devices containing nanogap electrodes. Our finite element modeling suggests optimal device geometry that should allow simultaneous DNA detection. The design of sub$10 \mathrm{~nm}$ electrodes at wafer scale is suitable not only for nanopore related experiments but as well for use in nextgeneration modern biosensors based on either plasmonics ${ }^{26}$ or nanoelectronics detection mechanism. ${ }^{27}$

\section{ASSOCIATED CONTENT}

\section{S Supporting Information}

Nine additional figures and information. This material is available free of charge via the Internet at http://pubs.acs.org.

\section{AUTHOR INFORMATION}

\section{Corresponding Authors}

*E-mail: (A.R.) aleksandra.radenovic@epfl.ch.

*E-mail: (L.F.) laszlo.forro@epfl.ch.

\section{Author Contributions}

A.F., F.T., A.M., L.F., and A.R. designed the study. A.F. and F.T. fabricated nanogap-nanopores, carried out TEM and SEM nanopore characterization, and performed nanopore sensing measurements presented in the paper and Supporting Information. A.R. built the nanopore sensing setup. A.F. and F.T. analyzed data presented in the paper and Supporting Information. S.K. performed COMSOL modeling presented in the paper and Supporting Information. A.F., L.F., and A.R. prepared the manuscript. L.F., A.M., and A.R. supervised the project. All the authors read and commented on the manuscript.

\section{Notes}

The authors declare no competing financial interest.

\section{ACKNOWLEDGMENTS}

This work was financially supported by FP7 nanoDNA sequencing grant. We thank the Centre Interdisciplinaire de Microscopie Electronique (CIME) at EPFL for access to electron microscopes; special thanks to D. T. L. Alexander for providing training and technical assistance with TEM. We thank Dr. Sudhir Husale for initial work on nanogap devices. Devices fabrication was partially carried out at the EPFL Center for Micro/Nanotechnology (CMi).

\section{REFERENCES}

(1) Lagerqvist, J.; Zwolak, M.; Di Ventra, M. Nano Lett. 2006, 6 (4), 779-782.

(2) Zwolak, M.; Di Ventra, M. Rev. Mod. Phys. 2008, 80 (1), 141165.

(3) Schneider, G. F.; Kowalczyk, S. W.; Calado, V. E.; Pandraud, G.; Zandbergen, H. W.; Vandersypen, L. M. K.; Dekker, C. Nano Lett. 2010, 10 (8), 3163-3167.

(4) Merchant, C. A.; Healy, K.; Wanunu, M.; Ray, V.; Peterman, N.; Bartel, J.; Fischbein, M. D.; Venta, K.; Luo, Z. T.; Johnson, A. T. C.; Drndic, M. Nano Lett. 2010, 10 (8), 2915-2921.

(5) Garaj, S.; Hubbard, W.; Reina, A.; Kong, J.; Branton, D.; Golovchenko, J. A. Nature 2010, 467 (7312), 190-U73.

(6) Tsutsui, M.; Taniguchi, M.; Yokota, K.; Kawai, T. Nat. Nanotechnol. 2010, 5 (4), 286-290.

(7) Tsutsui, M.; Rahong, S.; Iizumi, Y.; Okazaki, T.; Taniguchi, M.; Kawai, T. Sci. Rep. 2011, 1.

(8) Gierhart, B. C.; Flowitt, D. G.; Chen, S. J.; Zhu, Z.; Kotecki, D. E.; Smith, R. L.; Collins, S. D. Sens. Actuators, B 2008, 132 (2), $593-$ 600.

(9) Ivanov, A. P.; Instuli, E.; McGilvery, C. M.; Baldwin, G.; McComb, D. W.; Albrecht, T.; Edel, J. B. Nano Lett. 2011, 11 (1), 279-285.

(10) Healy, K.; Ray, V.; Willis, L. J.; Peterman, N.; Bartel, J.; Drndic, M. Electrophoresis 2012, 33 (23), 3488-3496.

(11) Radenovic, A.; Trepagnier, E.; Csencsits, R.; Downing, K. H.; Liphardt, J. Appl. Phys. Lett. 2008, 93, 18.

(12) Kowalczyk, S. W.; Grosberg, A. Y.; Rabin, Y.; Dekker, C. Nanotechnology 2011, 22, 315101.

(13) Chen, P.; Mitsui, T.; Farmer, D. B.; Golovchenko, J.; Gordon, R. G.; Branton, D. Nano Lett. 2004, 4 (7), 1333-1337.

(14) Smeets, R. M. M.; Dekker, N. H.; Dekker, C. Nanotechnology 2009, 20, 095501.

(15) Cervera, J.; Schiedt, B.; Ramirez, P. Europhys. Lett. 2005, 71 (1), $35-41$.

(16) Siwy, Z.; Kosinska, I. D.; Fulinski, A.; Martin, C. R. Phys. Rev. Lett. 2005, 94, 048102-1-048102-4.

(17) Karnik, R.; Duan, C. H.; Castelino, K.; Daiguji, H.; Majumdar, A. Nano Lett. 2007, 7 (3), 547-551.

(18) Ramirez, P.; Gomez, V.; Cervera, J.; Schiedt, B.; Mafe, S. J. Chem. Phys. 2007, 126, 194703.

(19) Kubeil, C.; Bund, A. J. Phys. Chem. C 2011, 115 (16), 78667873.

(20) Meller, A.; Nivon, L.; Branton, D. Phys. Rev. Lett. 2001, 86 (15), $3435-3438$

(21) Wanunu, M.; Morrison, W.; Rabin, Y.; Grosberg, A. Y.; Meller, A. Nat. Nanotechnol. 2010, 5 (2), 160-5.

(22) Meller, A.; Branton, D. Electrophoresis 2002, 23 (16), 25832591.

(23) Raillon, C.; Granjon, P.; Graf, M.; Steinbock, L. J.; Radenovic, A. Nanoscale 2012, 4 (16), 4916-24.

(24) Raillon, C.; Cousin, P.; Traversi, F.; Garcia-Cordero, E.; Hernandez, N.; Radenovic, A. Nano Lett. 2012, 12 (3), 1157-64.

(25) Manfrinato, V. R.; Zhang, L.; Su, D.; Duan, H.; Hobbs, R. G.; Stauch, E. A.; Berggren, K. K. Nano Lett. 2013, 13 (4), 1555-1558.

(26) Brolo, A. G. Nat. Photonics 2012, 6 (11), 709-713.

(27) Chen, J. R.; Miao, Y. Q.; He, N. Y.; Wu, X. H.; Li, S. J. Biotechnol. Adv. 2004, 22 (7), 505-518. 